\title{
Generalized Fractional Hadamard and Fejér-Hadamard Inequalities for Generalized Harmonically Convex Functions
}

\author{
Chahn Yong Jung, ${ }^{1}$ Muhammad Yussouf, ${ }^{2}$ Yu-Ming Chu $\left(\mathbb{D},{ }^{3,4}\right.$ Ghulam Farid (D), \\ and Shin Min Kang ${ }^{6}$ \\ ${ }^{1}$ Department of Business Administration, Gyeongsang National University, Jinju 52828, Republic of Korea \\ ${ }^{2}$ Department of Mathematics, University of Sargodha, Sargodha, Pakistan \\ ${ }^{3}$ Department of Mathematics, Huzhou University, Huzhou 313000, China \\ ${ }^{4}$ Hunan Provincial Key Laboratory of Mathematical Modeling and Analysis in Engineering, \\ Changsha University of Science \& Technology, Changsha 410114, China \\ ${ }^{5}$ Department of Mathematics, COMSATS University Islamabad, Attock, Pakistan \\ ${ }^{6}$ Center for General Education, China Medical University, Taichung 40402, Taiwan
}

Correspondence should be addressed to Yu-Ming Chu; chuyuming@zjhu.edu.cn and Ghulam Farid; faridphdsms@hotmail.com

Received 9 July 2020; Accepted 15 October 2020; Published 4 November 2020

Academic Editor: Sei Ichiro Ueki

Copyright (c) 2020 Chahn Yong Jung et al. This is an open access article distributed under the Creative Commons Attribution License, which permits unrestricted use, distribution, and reproduction in any medium, provided the original work is properly cited.

In this paper, we define a new function, namely, harmonically $(\alpha, h-m)$-convex function, which unifies various kinds of harmonically convex functions. Generalized versions of the Hadamard and the Fejér-Hadamard fractional integral inequalities for harmonically $(\alpha, h-m)$-convex functions via generalized fractional integral operators are proved. From presented results, a series of fractional integral inequalities can be obtained for harmonically convex, harmonically $(h-m)$-convex, harmonically $(\alpha, m)$-convex, and related functions and for already known fractional integral operators.

\section{Introduction and Preliminary Results}

Fractional integral inequalities are the generalizations and extensions of classical integral inequalities using fractional integral/derivative operators. There are many well-known inequalities which have been extended for fractional calculus operators: for example, Hadamard, Minkowski, Ostrowski, Grüss, Ostrowski-Grüss, and Chebyshev inequalities have been extensively studied in recent decades, see [1-8]. The aim of this paper is to present the Hadamard and the Fejér-Hadamard inequalities for fractional integral operators for harmonically $(\alpha, h-m)$-convex functions. We begin from the fractional integral operators defined by Andrić et al. in [2] containing an extended generalized Mittag-Leffler function in their kernels.

Definition 1 (see [2]). Let $\omega, \tau, \delta, \rho, c \in \mathbb{C}, \mathfrak{R}(\tau), \mathfrak{R}(\delta)>0$, and $\mathfrak{R}(c)>\mathfrak{R}(\rho)>0$ with $p \geq 0, \sigma, r>0$, and $0<k \leq r+\sigma$.
Let $f \in L_{1}[a, b]$ and $x \in[a, b]$. Then, the generalized fractional integral operators $\varepsilon_{\sigma, \tau, \delta, \omega, a^{+}}^{\rho, r, k} f$ and $\varepsilon_{\sigma, \tau, \delta, \omega, b^{-}}^{\rho, r, k}$ are defined as follows:

$$
\begin{aligned}
& \left(\varepsilon_{\sigma, \tau, \delta, \omega, a^{+}}^{\rho, r, k, c} f\right)(x ; p)=\int_{a}^{x}(x-t)^{\tau-1} E_{\sigma, \tau, \delta}^{\rho, r, k, c}\left(\omega(x-t)^{\sigma} ; p\right) f(t) \mathrm{d} t, \\
& \left(\varepsilon_{\sigma, \tau, \delta, \omega, b^{-}}^{\rho, r, k, c} f\right)(x ; p)=\int_{x}^{b}(t-x)^{\tau-1} E_{\sigma, \tau, \delta}^{\rho, r, k, c}\left(\omega(t-x)^{\sigma} ; p\right) f(t) \mathrm{d} t,
\end{aligned}
$$

where

$$
E_{\sigma, \tau, \delta}^{\rho, r, k, c}(t ; p)=\sum_{n=0}^{\infty} \frac{\beta_{p}(\rho+\mathrm{nk}, c-\rho)(c)_{\mathrm{nk}} t^{n}}{\beta(\rho, c-\rho) \Gamma(\sigma n+\tau)(\delta)_{\mathrm{nr}}},
$$

is the extended generalized Mittag-Leffler function.

In [9], Farid defined elegantly the unified integral operators as follows: 
Definition 2. Let $f, g:[a, b] \longrightarrow \mathbb{R}, 0<a<b$, be the functions such that $f$ is positive and $f \in L_{1}[a, b]$ and $g$ is differentiable and strictly increasing. Also, let $(\phi / x)$ be an increasing function on $[a, \infty)$ and $\omega, \tau, \delta, \rho, c \in \mathbb{C}$, $\mathfrak{R}(\tau), \mathfrak{R}(\delta)>0$, and $\mathfrak{R}(c)>\mathfrak{R}(\rho)>0$ with $p \geq 0, \sigma, r>0$, and $0<k \leq r+\sigma$. Then, for $x \in[a, b]$, the integral operators $\left({ }_{g} F_{\sigma, \tau, \delta, \omega, a^{+}}^{\phi, \rho, r, k, c} f\right)$ and $\left({ }_{g} F_{\sigma, \tau, \delta, \omega, b^{-}}^{\phi, \rho, r, k, c} f\right)$ are defined as follows:

$$
\begin{aligned}
\left({ }_{g} F_{\sigma, \tau, \delta, \omega, a^{+}}^{\phi, \rho, r, k, c} f\right)(x ; p)= & \int_{a}^{x} \frac{\phi(g(x)-g(t))}{g(x)-g(t)} \\
& \cdot E_{\sigma, \tau, \delta}^{\rho, r, k, c}\left(\omega(g(x)-g(t))^{\sigma} ; p\right) \\
& \cdot f(t) d(g(t)), \\
\left({ }_{g} F_{\sigma, \tau, \delta, \omega, b^{-}}^{\phi, \rho, r, k, c} f\right)(x ; p)= & \int_{x}^{b} \frac{\phi(g(t)-g(x))}{g(t)-g(x)} \\
& \cdot E_{\sigma, \tau, \delta}^{\rho, r, k, c}\left(\omega(g(t)-g(x))^{\sigma} ; p\right) \\
& \cdot f(t) d(g(t)) .
\end{aligned}
$$

The following definition of extended generalized fractional integral operators containing an extended MittagLeffler function in the kernel can be extracted by taking $\phi(x)=x^{\tau}$ in Definition 2 .

Definition 3. Let $f, g:[a, b] \longrightarrow \mathbb{R}, 0<a<b$, be the functions such that $f$ is positive and $f \in L_{1}[a, b]$ and $g$ is differentiable and strictly increasing. Also, let $\omega, \tau, \delta, \rho, c \in \mathbb{C}$, $\mathfrak{R}(\tau), \mathfrak{R}(\delta)>0$, and $\mathfrak{R}(c)>\mathfrak{R}(\rho)>0$ with $p \geq 0, \sigma, r>0$, and $0<k \leq r+\sigma$. Then, for $x \in[a, b]$, the generalized fractional integral operators are defined as follows:

$$
\begin{aligned}
\left({ }_{g} Y_{\sigma, \tau, \delta, \omega, a^{+}}^{\rho, r, k, c} f\right)(x ; p)= & \int_{a}^{x}(g(x)-g(t))^{\tau-1} \\
& \cdot E_{\sigma, \tau, \delta}^{\rho, r, k, c}\left(\omega(g(x)-g(t))^{\sigma} ; p\right) \\
& \cdot f(t) d(g(t)), \\
\left({ }_{g} Y_{\sigma, \tau, \delta, \omega, b^{-}}^{\rho, r, k, c} f\right)(x ; p)= & \int_{x}^{b}(g(t)-g(x))^{\tau-1} \\
& \cdot E_{\sigma, \tau, \delta}^{\rho, r, k, c}\left(\omega(g(t)-g(x))^{\sigma} ; p\right) \\
& \cdot f(t) d(g(t)) .
\end{aligned}
$$

Fractional integral operators (4) and (5) produce particularly various well-known fractional integral operators.

The Hadamard inequality gives the bounds of integral mean of a convex function on a closed interval. It is equivalently defined by the convex function. Convex functions have a significant role in the field of mathematical inequalities.

Definition 4. A function $f:[a, b] \longrightarrow \mathbb{R}$ is said to be convex if

$$
f(t x+(1-t) y) \leq t f(x)+(1-t) f(y),
$$

holds for all $x, y \in[a, b]$ and $t \in[0,1]$.
The Hadamard inequality is stated in the following theorem.

Theorem 1. Let $f:[a, b] \longrightarrow \mathbb{R}$ be a convex function. Then, the following inequality holds:

$$
f\left(\frac{a+b}{2}\right) \leq \frac{1}{b-a} \int_{a}^{b} f(x) \mathrm{d} x \leq \frac{f(a)+f(b)}{2} .
$$

The Fejér-Hadamard inequality proved by Fejér in [10] generalizes the Hadamard inequality, and it is given as follows:

Theorem 2. Let $f:[a, b] \longrightarrow \mathbb{R}$ be a convex function and $g:[a, b] \longrightarrow \mathbb{R}$ be nonnegative, integrable, and symmetric about $(a+b / 2)$. Then, the following inequality holds:

$$
\begin{aligned}
f\left(\frac{a+b}{2}\right) \int_{a}^{b} g(x) \mathrm{d} x & \leq \int_{a}^{b} f(x) g(x) \mathrm{d} x \\
& \leq \frac{f(a)+f(b)}{2} \int_{a}^{b} g(x) \mathrm{d} x .
\end{aligned}
$$

The definition of the convex function motivates the researchers to define new notions and their consequences. A notion of the harmonically convex function is defined in [11].

Definition 5. Let $I$ be an interval such that $I \subseteq \mathbb{R}_{+}$; a function $f: I \longrightarrow \mathbb{R}$ is said to be harmonically convex if

$$
f\left(\frac{a b}{t a+(1-t) b}\right) \leq t f(b)+(1-t) f(a),
$$

holds for all $a, b \in I$ and $t \in[0,1]$.

This notation is further extended to the harmonically $(\alpha, m)$-convex function in [12] and harmonically $h$-convex function in [13].

Definition 6 (see [12]). A function $f:[0, b] \longrightarrow \mathbb{R}, b>0$, is said to be harmonically $(\alpha, m)$-convex if $f$ is nonnegative and

$$
f\left(\frac{m x y}{m t y+(1-t) x}\right) \leq t^{\alpha} f(x)+m\left(1-t^{\alpha}\right) f(y),
$$

holds for all $x, y \in[0, b], t, \alpha \in[0,1]$, and $m \in(0,1]$.

Definition 7 (see [13]). Let $h:[0,1] \subseteq J \longrightarrow \mathbb{R}$ be a nonnegative function. A function $f: J \subseteq \mathbb{R}_{+} \longrightarrow \mathbb{R}$ is said to be harmonically $h$-convex if

$$
f\left(\frac{x y}{t y+(1-t) x}\right) \leq h(t) f(x)+h(1-t) f(y),
$$

holds for all $x, y \in J$ and $t \in(0,1)$.

In the upcoming section, we define a new generalized notion, namely, harmonically $(\alpha, h-m)$-convex function, which unifies all known definitions of harmonically convex 
functions. By using this new definition, we will prove Hadamard and Fejér-Hadamard inequalities for generalized fractional integral operators involving an extended generalized Mittag-Leffler function. Also, results for harmonically $(h-m)$-convex functions and harmonically $(\alpha, m)$-convex functions are deduced.

\section{Main Results}

First, we define the harmonically $(\alpha, h-m)$-convex function as follows:

Definition 8 . Let $h:[0,1] \subseteq J \longrightarrow \mathbb{R}$ be a nonnegative function. A function $f: J \subseteq \mathbb{R}_{+} \longrightarrow \mathbb{R}$ is said to be harmonically $(\alpha, h-m)$-convex if

$$
f\left(\frac{m x y}{m t y+(1-t) x}\right) \leq h\left(t^{\alpha}\right) f(x)+m h\left(1-t^{\alpha}\right) f(y),
$$

holds for all $x, y \in J, t, \alpha \in[0,1]$, and $m \in(0,1]$.

It unifies the definitions of harmonically $(\alpha, m)$-convexity and harmonically $h$-convexity of functions. For different specific choices of $\alpha, h$, and $m$, almost all kinds of well-known harmonically convex functions can be obtained, see the remark as follows.

\section{Remark 1}

(i) If $h(t)=t$, then a harmonically $(\alpha, m)$-convex function can be obtained [12]

(ii) If $\alpha=1$ and $h(t)=t^{s}$, then a harmonically $(s, m)$-convex function can be obtained [14]

(iii) If $\alpha=1$ and $h(t)=t$, then a harmonically $m$-convex function can be obtained [14]

(iv) If $\alpha=h(t)=m=1$, then a harmonically $P$-function can be obtained [13]

(v) If $\alpha=1, h(t)=t^{s}$, and $m=1$, then a harmonically $s$-convex function can be obtained [13] (vi) If $\alpha=1, h(t)=(1 / t)$, and $m=1$, then a harmonically Godunova-Levin function can be obtained [13]

(vii) If $\alpha=1, h(t)=\left(1 / t^{s}\right)$, and $m=1$, then a harmonically $s$-Godunova-Levin function can be obtained [13]

(viii) If we set $m=1$ and $\alpha=1$, then a harmonically $h$-convex function can be achieved [13]

(ix) By putting $\alpha=1, h(t)=t$, and $m=1$, then a harmonically convex function can be obtained [11]

We give another new notion of the harmonically $(h-m)$-convex function by setting $\alpha=1$ in Definition 8 as follows.

Definition 9. Let $h:[0,1] \subseteq J \longrightarrow \mathbb{R}$ be a nonnegative function. A function $f: J \subseteq \mathbb{R}_{+} \longrightarrow \mathbb{R}$ is said to be harmonically $(h-m)$-convex if

$$
f\left(\frac{m x y}{m t y+(1-t) x}\right) \leq h(t) f(x)+m h(1-t) f(y) .
$$

Now, we apply Definition 8 and operators (4) and (5) to find the Hadamard and Fejér-Hadamard inequalities.

In the whole paper, we have used the following notations frequently:

$$
\begin{aligned}
& \left(\mathscr{F}_{b, \tau}^{a^{+}}\right)(\omega, f)=\left({ }_{g} \Upsilon_{\sigma, \tau, \delta, \omega, a^{+}}^{\rho, r, k, c} f\right)(b ; p), \\
& \left(\mathscr{F}_{a, \tau}^{b^{-}}\right)(\omega, f)=\left({ }_{g} \Upsilon_{\sigma, \tau, \delta, \omega, b^{-}}^{\rho, r, k, c} f\right)(a ; p) .
\end{aligned}
$$

Theorem 3. Let $f, g:[a, b] \longrightarrow \mathbb{R}, 0<a<b$, range $(g) \subset[a, b]$, be the functions such that $f$ is positive and $f \in L_{1}[a, b]$ and $g$ is differentiable and strictly increasing. If $f$ is harmonically $(\alpha, h-m)$-convex on $[a, b]$, then for operators (4) and (5), we have

$$
\begin{aligned}
f\left(\frac{2 m^{2} g(a) g(b)}{g(a)+m g(b)}\right)\left(\mathscr{F}_{g^{-1}\left(1 / m^{2} g(b)\right), \tau}^{g^{-1}(1 / m g(a))^{-}}\right)\left(m^{\sigma} \omega^{\prime}, 1\right) \leq & h\left(\frac{1}{2^{\alpha}}\right)\left(\mathscr{F}_{g^{-1}\left(1 / m^{2} g(b)\right), \tau}^{g^{-1}((1 / m g(a))-}\right)\left(m^{\sigma} \omega^{\prime}, f \circ \psi\right) \\
& +m^{1-2 \tau} h\left(1-\frac{1}{2^{\alpha}}\right)\left(\mathscr{F}_{g^{-1}(1 / g(a)), \tau}^{g^{-1}(1 / m g(b))^{+}}\right)\left(\omega^{\prime}, f \circ \psi\right) \leq\left(\frac{m g(b)-g(a)}{m^{2} g(a) g(b)}\right), \\
& +\left\{m\left(h\left(\frac{1}{2^{\alpha}}\right) f\left(\frac{g(a)}{m^{2}}\right)+m h\left(1-\frac{1}{2^{\alpha}}\right) f(g(b))\right) \int_{0}^{1} t^{\tau-1} E_{\sigma, \tau, \delta}^{\rho, r, k, c}\left(\omega\left(t^{\sigma}\right) ; p\right) h\left(t^{\alpha}\right) \mathrm{d} t\right. \\
& \left.+\left(h\left(\frac{1}{2^{\alpha}}\right) f(g(b))+m h\left(1-\frac{1}{2^{\alpha}}\right) f(g(a))\right) \int_{0}^{1} t^{\tau-1} E_{\sigma, \tau, \delta}^{\rho, r, k, c}\left(\omega\left(t^{\sigma}\right) ; p\right) h\left(1-t^{\alpha}\right) \mathrm{d} t\right\},
\end{aligned}
$$

where $\omega^{\prime}=\omega(m g(a)(b) / m g(b)-g(a))^{\sigma}$ and $\psi(t)=$ $(1 / g(t))$ for all $t \in[(1 / b),(1 / a)]$.
Proof. Since $f$ is harmonically $(\alpha, h-m)$-convex, for all $x, y \in[a, b]$, the following inequality holds: 


$$
f\left(\frac{2 m g(x) g(y)}{m g(y)+g(x)}\right) \leq h\left(\frac{1}{2^{\alpha}}\right) f(g(x))+m h\left(1-\frac{1}{2^{\alpha}}\right) f(g(y)) .
$$

By setting $\quad g(x)=(m g(a) g(b) / t g(b)+(1-t)$ $(g(a) / m))$ and $g(y)=(m g(a) g(b) / t g(a)+m(1-t) g(b))$ in (16), we have

$$
f\left(\frac{2 m^{2} g(a) g(b)}{g(a)+m g(b)}\right) \leq h\left(\frac{1}{2^{\alpha}}\right) f\left(\frac{m g(a) g(b)}{\operatorname{tg}(b)+(1-t)(g(a) / m)}\right)+m h\left(1-\frac{1}{2^{\alpha}}\right) f\left(\frac{m g(a) g(b)}{\operatorname{tg}(a)+m(1-t) g(b)}\right) .
$$

Multiplying (17) by $t^{\tau-1} E_{\sigma, \tau, \delta}^{\rho, r, k, c}\left(\omega\left(t^{\sigma}\right) ; p\right)$ and integrating over $[0,1]$, we get

$$
\begin{aligned}
& f\left(\frac{2 m^{2} g(a) g(b)}{g(a)+m g(b)}\right) \int_{0}^{1} t^{\tau-1} E_{\sigma, \tau, \delta}^{\rho, r, k, c}\left(\omega t^{\sigma} ; p\right) \mathrm{d} t \\
& \leq h\left(\frac{1}{2^{\alpha}}\right) \int_{0}^{1} t^{\tau-1} E_{\sigma, \tau, \delta}^{\rho, r, k, c}\left(\omega t^{\sigma} ; p\right) f\left(\frac{m g(a) g(b)}{t g(b)+(1-t)(g(a) / m)}\right) \mathrm{d} t \\
& \quad+m h\left(1-\frac{1}{2^{\alpha}}\right) \int_{0}^{1} t^{\tau-1} E_{\sigma, \tau, \delta}^{\rho, r, k, c}\left(\omega t^{\sigma} ; p\right) f\left(\frac{m g(a) g(b)}{\operatorname{tg}(a)+m(1-t) g(b)}\right) \mathrm{d} t .
\end{aligned}
$$

By setting $g(x)=(\operatorname{tg}(b)+(1-t)(g(a) / m) / m g(a) g(b))$ and $g(y)=(t g(a)+m(1-t) g(b) / m g(a) g(b))$ in (18) and using (4) and (5), the first inequality of (15) can be obtained.
On the contrary, from the harmonically $(\alpha, h-m)$-convexity of $f$, we have

$$
\begin{aligned}
h\left(\frac{1}{2^{\alpha}}\right) & f\left(\frac{m g(a) g(b)}{\operatorname{tg}(b)+(1-t)(g(a) / m)}\right)+m h\left(1-\frac{1}{2^{\alpha}}\right) f\left(\frac{m g(a) g(b)}{\operatorname{tg}(a)+m(1-t) g(b)}\right) \\
\leq & h\left(\frac{1}{2^{\alpha}}\right)\left(h\left(1-t^{\alpha}\right) f(g(b))+m h\left(t^{\alpha}\right) f\left(\frac{g(a)}{m^{2}}\right)\right) \\
& +m h\left(1-\frac{1}{2^{\alpha}}\right)\left(h\left(1-t^{\alpha}\right) f(g(a))+m h\left(t^{\alpha}\right) f(g(b))\right) \\
= & m\left(h\left(\frac{1}{2^{\alpha}}\right) f\left(\frac{g(a)}{m^{2}}\right)+m h\left(1-\frac{1}{2^{\alpha}}\right) f(g(b))\right) h\left(t^{\alpha}\right) \\
& +\left(h\left(\frac{1}{2^{\alpha}}\right) f(g(b))+m h\left(1-\frac{1}{2^{\alpha}}\right) f(g(a))\right) h\left(1-t^{\alpha}\right) .
\end{aligned}
$$

Multiplying (19) by $t^{\tau-1} E_{\sigma, \tau, \delta}^{\rho, r, k, c}\left(\omega\left(t^{\sigma}\right) ; p\right)$ and then integrating over $[0,1]$, we get

$$
\begin{aligned}
& h\left(\frac{1}{2^{\alpha}}\right) \int_{0}^{1} t^{\tau-1} E_{\sigma, \tau, \delta}^{\rho, r, k, c}\left(\omega t^{\sigma} ; p\right) f\left(\frac{m g(a) g(b)}{t g(b)+(1-t)(g(a) / m)}\right) \mathrm{d} t \\
& \quad+m h\left(1-\frac{1}{2^{\alpha}}\right) \int_{0}^{1} t^{\tau-1} E_{\sigma, \tau, \delta}^{\rho, r, k, c}\left(\omega t^{\sigma} ; p\right) f\left(\frac{m g(a) g(b)}{\operatorname{tg}(a)+m(1-t) g(b)}\right) \mathrm{d} t \\
& \leq m\left(h\left(\frac{1}{2^{\alpha}}\right) f\left(\frac{g(a)}{m^{2}}\right)+m h\left(1-\frac{1}{2^{\alpha}}\right) f(g(b))\right) \int_{0}^{1} t^{\tau-1} E_{\sigma, \tau, \delta}^{\rho, r, k, c}\left(\omega t^{\sigma} ; p\right) h\left(t^{\alpha}\right) \mathrm{d} t \\
& \quad+\left(h\left(\frac{1}{2^{\alpha}}\right) f(g(b))+m h\left(1-\frac{1}{2^{\alpha}}\right) f(g(a))\right) \int_{0}^{1} t^{\tau-1} E_{\sigma, \tau, \delta}^{\rho, r, k, c}\left(\omega t^{\sigma} ; p\right) h\left(t^{\alpha}\right) h\left(1-t^{\alpha}\right) \mathrm{d} t .
\end{aligned}
$$


By setting $g(x)=(\operatorname{tg}(b)+(1-t)(g(a) / m) / m g(a) g(b))$ and $g(y)=(\operatorname{tg}(a)+m(1-t) g(b) / m g(a) g(b))$ in $(20)$ and using (4) and (5), the second inequality of (15) can be obtained.

\section{Remark 2}

(i) By setting $p=0, \alpha=m=1, h(t)=t$, and $g=I$, Theorem 3.1 of [15] is obtained

(ii) By setting $\alpha=m=1, h(t)=t$, and $g=I$, Theorem 2.1 of [16] is obtained (iii) By setting $\omega=p=0, \alpha=m=1, h(t)=t$, and $g=I$, Theorem 4 of [6] is obtained

Theorem 4. Let $f, g, \hbar:[a, b] \longrightarrow \mathbb{R}, 0<a<b$, range $(g)$, range $(\hbar) \subset[a, b]$, be the functions such that $f$ is positive and $f \in L_{1}[a, b], g$ is differentiable and strictly increasing, and $\hbar$ is nonnegative and integrable. If $f$ is harmonically $(\alpha, h-m)$-convex and $f(1 / g(x))=f(1 /(1 / g(a))+$ $(1 / m g(b))-m g(x))$, then for operators (4) and (5), we have

$$
\begin{aligned}
& f\left(\frac{2 m^{2} g(a) g(b)}{g(a)+m g(b)}\right)\left(\mathscr{F}_{g^{-1}\left(1 / m^{2} g(b)\right), \tau}^{g^{-1}(1 / m g(a)-}\right)\left(m^{\sigma} \omega^{\prime}, \hbar \circ \psi\right) \\
& \leq\left(h\left(\frac{1}{2^{\alpha}}\right)+m h\left(1-\frac{1}{2^{\alpha}}\right)\right)\left(\begin{array}{c}
\mathscr{F}^{-1}(1 / m g(a))^{-} \\
g^{-1}\left(1 / m^{2} g(b)\right), \tau
\end{array}\right)\left(m^{\sigma} \omega^{\prime},(\hbar \circ \psi)(f \circ \psi)\right) \\
& \leq\left(\frac{m g(b)-g(a)}{m^{2} g(a) g(b)}\right)^{\tau}\left\{m\left(h\left(\frac{1}{2^{\alpha}}\right) f\left(\frac{g(a)}{m^{2}}\right)+m h\left(1-\frac{1}{2^{\alpha}}\right) f(g(b))\right)\right. \\
& \times \int_{0}^{1} t^{\tau-1} E_{\sigma, \tau, \delta}^{\rho, r, k, c}\left(\omega\left(t^{\sigma}\right) ; p\right) \hbar\left(\frac{m g(a) g(b)}{\operatorname{tg}(b)+(1-t)(g(a) / m)}\right) h\left(t^{\alpha}\right) \mathrm{d} t \\
& +\left(h\left(\frac{1}{2^{\alpha}}\right) f(g(b))+m h\left(1-\frac{1}{2^{\alpha}}\right) f(g(a))\right) \\
& \left.\times \int_{0}^{1} t^{\tau-1} E_{\sigma, \tau, \delta}^{\rho, r, k, c}\left(\omega\left(t^{\sigma}\right) ; p\right) \hbar\left(\frac{m g(a) g(b)}{\operatorname{tg}(b)+(1-t)(g(a) / m)}\right) h\left(1-t^{\alpha}\right) \mathrm{d} t\right\},
\end{aligned}
$$

where $\omega^{\prime}=\omega(m g(a)(b) / m g(b)-g(a))^{\sigma}$ and $\psi(t)=$ $(1 / g(t))$ for all $t \in[(1 / b),(1 / a)]$.
Proof. Multiplying (17) by $t^{\tau-1} \hbar((m g(a) g(b) / \operatorname{tg}(b)+(1-$ t) $(g(a) / m))) E_{\sigma, \tau, \delta}^{\rho, r, k, c}\left(\omega\left(t^{\sigma}\right) ; p\right)$ and then integrating over $[0,1]$, we get

$$
\begin{aligned}
& f\left(\frac{2 m^{2} g(a) g(b)}{g(a)+m g(b)}\right) \int_{0}^{1} t^{\tau-1} E_{\sigma, \tau, \delta}^{\rho, r, k, c}\left(\omega t^{\sigma} ; p\right) \hbar\left(\frac{m g(a) g(b)}{\operatorname{tg}(b)+(1-t)(g(a) / m)}\right) \mathrm{d} t \\
& \quad \leq h\left(\frac{1}{2^{\alpha}}\right) \int_{0}^{1} t^{\tau-1} E_{\sigma, \tau, \delta}^{\rho, r, k, c}\left(\omega t^{\sigma} ; p\right) \hbar\left(\frac{m g(a) g(b)}{\operatorname{tg}(b)+(1-t)(g(a) / m)}\right) f\left(\frac{m g(a) g(b)}{\operatorname{tg}(b)+(1-t)(g(a) / m)}\right) \mathrm{d} t \\
& \quad+m h\left(1-\frac{1}{2^{\alpha}}\right) \int_{0}^{1} t^{\tau-1} E_{\sigma, \tau, \delta}^{\rho, r, k, c}\left(\omega t^{\sigma} ; p\right) \hbar\left(\frac{m g(a) g(b)}{\operatorname{tg}(b)+(1-t)(g(a) / m)}\right) f\left(\frac{m g(a) g(b)}{\operatorname{tg}(a)+m(1-t) g(b)}\right) \mathrm{d} t .
\end{aligned}
$$

By choosing $g(x)=(\operatorname{tg}(b)+(1-t)(g(a) / m) / m g(a)$ $g(b))$, that is, $(m g(a) g(b) / \operatorname{tg}(a)+m(1-t) \quad g(b))=$ $(1 /(1 / g(a))(1 / m g(b))-m g(x))$, in (22) and using (4) and (5) and the condition $f(1 / g(x))=f(1 /(1 / g(a))$
$(1 / m g(b))-m g(x))$, the first inequality of (21) can be obtained. For the second inequality of (21), multiplying (19) by $t^{\tau-1} E_{\sigma, \tau, \delta}^{\rho, r, c}\left(\omega t^{\sigma} ; p\right) \hbar(m g(a) g(b) / \operatorname{tg}(b)+(1-t)(g(a) /$ $m)$ ) and then integrating over $[0,1]$, we get 


$$
\begin{aligned}
& h\left(\frac{1}{2^{\alpha}}\right) \int_{0}^{1} t^{\tau-1} E_{\sigma, \tau, \delta}^{\rho, r, c}\left(\omega t^{\sigma} ; p\right) \hbar\left(\frac{m g(a) g(b)}{\operatorname{tg}(b)+(1-t)(g(a) / m)}\right) f\left(\frac{m g(a) g(b)}{\operatorname{tg}(b)+(1-t)(g(a) / m)}\right) \mathrm{d} t \\
& \quad+m h\left(1-\frac{1}{2^{\alpha}}\right) \int_{0}^{1} t^{\tau-1} E_{\sigma, \tau, \delta}^{\rho, r, k, c}\left(\omega t^{\sigma} ; p\right) \hbar\left(\frac{m g(a) g(b)}{\operatorname{tg}(b)+(1-t)(g(a) / m)}\right) f\left(\frac{m g(a) g(b)}{\operatorname{tg}(a)+m(1-t) g(b)}\right) \mathrm{d} t \\
& \leq \quad m\left(h\left(\frac{1}{2^{\alpha}}\right) f\left(\frac{g(a)}{m^{2}}\right)+m h\left(1-\frac{1}{2^{\alpha}}\right) f(g(b))\right) \\
& \quad \cdot \int_{0}^{1} t^{\tau-1} E_{\sigma, \tau, \delta}^{\rho, r, k, c}\left(\omega t^{\sigma} ; p\right) \times \hbar\left(\frac{m g(a) g(b)}{\operatorname{tg}(b)+(1-t)(g(a) / m)}\right) h\left(t^{\alpha}\right) \mathrm{d} t \\
& \quad+\left(h\left(\frac{1}{2^{\alpha}}\right) f(g(b))+m h\left(1-\frac{1}{2^{\alpha}}\right) f(g(a))\right) \\
& \quad \times \int_{0}^{1} t^{\tau-1} E_{\sigma, \tau, \delta}^{\rho, r, k}\left(\omega t^{\sigma} ; p\right) h\left(t^{\alpha}\right) \hbar\left(\frac{m g(a) g(b)}{\operatorname{tg}(b)+(1-t)(g(a) / m)}\right) h\left(1-t^{\alpha}\right) \mathrm{d} t .
\end{aligned}
$$

By setting $g(x)=(\operatorname{tg}(b)+(1-t)(g(a) / m) / m g(a) g(b))$ in (23) and using (4) and (5) and the condition $f(1 / g(x))=f(1 /(1 / g(a))(1 / m g(b))-m g(x))$, the second inequality of (21) can be obtained.

\section{Remark 3}

(i) By setting $p=0, \alpha=m=1, h(t)=t \hbar(x)=1$, and $g=I$, Theorem 3.1 of [15] is obtained

(ii) By setting $g=I, \alpha=m=1, h(t)=t$, and $\hbar(x)=1$, Theorem 2.1 of [16] is obtained

(iii) By setting $\omega=p=0, \hbar(x)=1, \alpha=m=1, h(t)=t$, and $g=I$, Theorem 4 of [6] is obtained (iv) By setting $\omega=p=0, \tau=1, \alpha=m=1, h(t)=t$, and $g=I$, Theorem 8 of [4] is obtained

(v) By setting $\omega=p=0, \tau=1, \hbar(x)=1, \alpha=m=1$, $h(t)=t$, and $g=I$, Theorem 4 of [17] is obtained
Theorem 5. Let $f, g:[a, b] \longrightarrow \mathbb{R}, \quad 0<a<b$, range $(g) \subset[a, b]$, be the functions such that $f$ is positive and $f \in L_{1}[a, b]$ and $g$ is differentiable and strictly increasing. If $f$ is harmonically $(\alpha, h-m)$-convex on $[a, b]$, then for operators (4) and (5), we have

$$
\begin{aligned}
f( & \left.\frac{2 m^{2} g(a) g(b)}{g(a)+m g(b)}\right)\left(\mathscr{F}_{g^{-1}\left(1 / m^{2} g(b)\right), \tau}^{\left.g^{-1}(g)+m g(b) / 2 m^{2} g(a) g(b)\right)^{-}}\right)\left((2 m)^{\sigma} \omega^{\prime}, 1\right) \\
\leq & h\left(\frac{1}{2^{\alpha}}\right)\left(\mathscr{F}_{g^{-1}\left(1 / m^{2} g(b)\right), \tau}^{g^{-1}\left(g(a)+m g(b) / 2 m^{2} g(a) g(b)\right)^{-}}\right)\left((2 m)^{\sigma} \omega^{\prime}, f \circ \psi\right) \\
& +m^{1-2 \tau} h\left(1-\frac{1}{2^{\alpha}}\right)\left(\mathscr{F}_{g^{-1}(1 / g(a)), \tau}^{g^{-1}(g(a)+m g(b) / 2 m g(a) g(b))^{+}}\right)\left(2^{\sigma} \omega^{\prime}, f \circ \psi\right) \\
\leq & \left(\frac{m g(b)-g(a)}{2 m^{2} g(a) g(b)}\right)^{\tau}\left\{m\left(h\left(\frac{1}{2^{\alpha}}\right) f\left(\frac{g(a)}{m^{2}}\right)+m h\left(1-\frac{1}{2^{\alpha}}\right) f(g(b))\right)\right. \\
& \times \int_{0}^{1} t^{\tau-1} E_{\sigma, \tau, \delta}^{\rho, r, k, c}\left(\omega\left(t^{\sigma}\right) ; p\right) h\left(\left(\frac{t}{2}\right)^{\alpha}\right) \mathrm{d} t+\left(h\left(\frac{1}{2^{\alpha}}\right) f(g(b))+m h\left(1-\frac{1}{2^{\alpha}}\right) f(g(a))\right) \\
& \left.\times \int_{0}^{1} t^{\tau-1} E_{\sigma, \tau, \delta}^{\rho, r, k, c}\left(\omega\left(t^{\sigma}\right) ; p\right) h\left(1-\left(\frac{t}{2}\right)^{\alpha}\right) \mathrm{d} t\right\}, \\
\omega^{\prime}=\omega( & \left.\frac{m g(a)(b)}{m g(b)-g(a)}\right)^{\sigma},
\end{aligned}
$$

where $\psi(t)=(1 / g(t))$ and $t \in[(1 / b),(1 / a)]$. 
Proof. By setting $g(x)=(m g(a) g(b) /(t / 2) g(b)+(1-(t / 2))$

$(g(a) / m))$ and $g(y)=(m g(a) g(b) /(t / 2) g(a)+m(1-$

$(t / 2)) g(b))$ in (16), we have

$$
\begin{aligned}
f\left(\frac{2 m^{2} g(a) g(b)}{g(a)+m g(b)}\right) \leq & h\left(\frac{1}{2^{\alpha}}\right) f\left(\frac{m g(a) g(b)}{(t / 2) g(b)+(1-(t / 2))(g(a) / m)}\right) \\
& +m h\left(1-\frac{1}{2^{\alpha}}\right) f\left(\frac{m g(a) g(b)}{(t / 2) g(a)+m(1-(t / 2)) g(b)}\right) .
\end{aligned}
$$

Multiplying (25) by $t^{\tau-1} E_{\sigma, \tau, \delta}^{\rho, r, k, c}\left(\omega t^{\sigma} ; p\right)$ and then integrating over $[0,1]$, we get

$$
\begin{aligned}
& f\left(\frac{2 m^{2} g(a) g(b)}{g(a)+m g(b)}\right) \int_{0}^{1} t^{\tau-1} E_{\sigma, \tau, \delta}^{\rho, r, k, c}\left(\omega t^{\sigma} ; p\right) \mathrm{d} t \\
& \quad \leq h\left(\frac{1}{2^{\alpha}}\right) \int_{0}^{1} t^{\tau-1} E_{\sigma, \tau, \delta}^{\rho, r, k, c}\left(\omega t^{\sigma} ; p\right) f\left(\frac{m g(a) g(b)}{(t / 2) g(b)+(1-(t / 2))(g(a) / m)}\right) \mathrm{d} t \\
& \quad+m h\left(1-\frac{1}{2^{\alpha}}\right) \int_{0}^{1} t^{\tau-1} E_{\sigma, \tau, \delta}^{\rho, r, k, c}\left(\omega t^{\sigma} ; p\right) f\left(\frac{m g(a) g(b)}{(t / 2) g(a)+m(1-(t / 2)) g(b)}\right) \mathrm{d} t .
\end{aligned}
$$

By setting $g(x)=((t / 2) g(b)+(1-(t / 2))(g(a) / m) /$ $m g(a) g(b))$ and $g(y)=((t / 2) g(a)+m(1-(t / 2)) g(b) /$ $m g(a) g(b))$ in (26) and using (4) and (5), the first inequality of (24) can be obtained. On the contrary, from the harmonically $(\alpha, h-m)$-convexity of $f$, we have

$$
\begin{aligned}
& h\left(\frac{1}{2^{\alpha}}\right) f\left(\frac{m g(a) g(b)}{(t / 2) g(b)+(1-(t / 2))(g(a) / m)}\right)+m h\left(1-\frac{1}{2^{\alpha}}\right) f\left(\frac{m g(a) g(b)}{(t / 2) g(a)+m(1-(t / 2)) g(b)}\right) \\
& \leq h\left(\frac{1}{2^{\alpha}}\right)\left(h\left(1-\left(\frac{t}{2}\right)^{\alpha}\right) f(g(b))+m h\left(\left(\frac{t}{2}\right)^{\alpha}\right) f\left(\frac{g(a)}{m^{2}}\right)\right) \\
& \quad+m h\left(1-\frac{1}{2^{\alpha}}\right)\left(h\left(1-\left(\frac{t}{2}\right)^{\alpha}\right) f(g(a))+m h\left(\left(\frac{t}{2}\right)^{\alpha}\right) f(g(b))\right) \\
& =m\left(h\left(\frac{1}{2^{\alpha}}\right) f\left(\frac{g(a)}{m^{2}}\right)+m h\left(1-\frac{1}{2^{\alpha}}\right) f(g(b))\right) h\left(\left(\frac{t}{2}\right)^{\alpha}\right) \\
& +\left(h\left(\frac{1}{2^{\alpha}}\right) f(g(b))+m h\left(1-\frac{1}{2^{\alpha}}\right) f(g(a))\right) h\left(1-\left(\frac{t}{2}\right)^{\alpha}\right) .
\end{aligned}
$$

Multiplying (27) by $t^{\tau-1} E_{\sigma, \tau, \delta}^{\rho, r, k, c}\left(\omega t^{\sigma} ; p\right)$ and integrating over $[0,1]$, we get 


$$
\begin{aligned}
& h\left(\frac{1}{2^{\alpha}}\right) \int_{0}^{1} t^{\tau-1} E_{\sigma, \tau, \delta}^{\rho, r, k, c}\left(\omega t^{\sigma} ; p\right) f\left(\frac{m g(a) g(b)}{(t / 2) g(b)+(1-(t / 2))(g(a) / m)}\right) \mathrm{d} t \\
& +m h\left(1-\frac{1}{2^{\alpha}}\right) \int_{0}^{1} t^{\tau-1} E_{\sigma, \tau, \delta}^{\rho, r, k, c}\left(\omega t^{\sigma} ; p\right) f\left(\frac{m g(a) g(b)}{(t / 2) g(a)+m(1-(t / 2)) g(b)}\right) \mathrm{d} t \\
& \leq \quad m\left(h\left(\frac{1}{2^{\alpha}}\right) f\left(\frac{g(a)}{m^{2}}\right)+m h\left(1-\frac{1}{2^{\alpha}}\right) f(g(b))\right) \\
& \quad \times \int_{0}^{1} t^{\tau-1} E_{\sigma, \tau, \delta}^{\rho, r, k, c}\left(\omega t^{\sigma} ; p\right) h\left(\left(\frac{t}{2}\right)^{\alpha}\right) \mathrm{d} t+\left(h\left(\frac{1}{2^{\alpha}}\right) f(g(b))+m h\left(1-\frac{1}{2^{\alpha}}\right) f(g(a))\right) \\
& \quad \times \int_{0}^{1} t^{\tau-1} E_{\sigma, \tau, \delta}^{\rho, r, k, c}\left(\omega t^{\sigma} ; p\right) h\left(1-\left(\frac{t}{2}\right)^{\alpha}\right) \mathrm{d} t .
\end{aligned}
$$

By setting $g(x)=((t / 2) g(b)+(1-(t / 2))(g(a) / m) /$ $m g(a) g(b))$ and $g(y)=((t / 2) g(a)+m(1-(t / 2)) g(b) /$ $m g(a) g(b))$ in (28) and using (4) and (5), the second inequality of (24) can be obtained.

\section{Remark 4}

(i) By setting $p=0, \alpha=m=1, h(t)=t$, and $g=I$, Theorem 3.3 of [15] is obtained (ii) By setting $\alpha=m=1, h(t)=t$, and $g=I$, Theorem 2.3 of [16] is obtained

Theorem 6. Let $f, g, \hbar:[a, b] \longrightarrow \mathbb{R}, 0<a<b$, range $(g)$, range $(\hbar) \subset[a, b]$, be the functions such that $f$ is positive and $f \in L_{1}[a, b], g$ is differentiable and strictly increasing, and $\hbar$ is nonnegative and integrable. If $f$ is harmonically $(\alpha, h-m)$ convex and $f(1 / g(x))=f(1 /(1 / g(a))(1 / m g(b))-$ $m g(x))$, then for operators (4) and (5), we have

$$
\begin{aligned}
f( & \left.\frac{2 m^{2} g(a) g(b)}{g(a)+m g(b)}\right)\left(\mathscr{F}_{g^{-1}\left(1 / m^{2} g(b)\right), \tau}^{g^{-1}\left(g(a)+m g(b) / 2 m^{2} g(a) g(b)\right)^{-}}\right)\left((2 m)^{\sigma} \omega^{\prime}, \hbar \circ \psi\right) \\
\leq & \left(h\left(\frac{1}{2^{\alpha}}\right)+m h\left(1-\frac{1}{2^{\alpha}}\right)\right)\left(\mathscr{F}_{g^{-1}\left(1 / m^{2} g(b)\right), \tau}^{g^{-1}\left(g(a) m g(b) / 2 m^{2} g(a) g(b)\right)^{-}}\right)\left((2 m)^{\sigma} \omega^{\prime},(\hbar \circ \psi)(f \circ \psi)\right) \\
\leq & \left(\frac{m g(b)-g(a)}{2 m^{2} g(a) g(b)}\right)^{\tau}\left\{m\left(h\left(\frac{1}{2^{\alpha}}\right) f\left(\frac{g(a)}{m^{2}}\right)+m h\left(1-\frac{1}{2^{\alpha}}\right) f(g(b))\right)\right. \\
& \times \int_{0}^{1} t^{\tau-1} E_{\sigma, \tau, \delta}^{\rho, r, k, c}\left(\omega\left(t^{\sigma}\right) ; p\right) \hbar\left(\frac{m g(a) g(b)}{(t / 2) g(b)+(1-(t / 2))(g(a) / m)}\right) h\left(\left(\frac{t}{2}\right)^{\alpha}\right) \mathrm{d} t \\
& +\left(h\left(\frac{1}{2^{\alpha}}\right) f(g(b))+m h\left(1-\frac{1}{2^{\alpha}}\right) f(g(a))\right) \\
\quad & \left.\int_{0}^{1} t^{\tau-1} E_{\sigma, \tau, \delta}^{\rho, r, k, c}\left(\omega\left(t^{\sigma}\right) ; p\right) \hbar\left(\frac{m g(a) g(b)}{(t / 2) g(b)+(1-(t / 2))(g(a) / m)}\right) h\left(1-\left(\frac{t}{2}\right)^{\alpha}\right) \mathrm{d} t\right\},
\end{aligned}
$$

where $\quad \omega^{\prime}=\omega(m g(a)(b) / m g(b)-g(a))^{\sigma}$ $\psi(t)=(1 / g(t))$ for all $t \in[(1 / b),(1 / a)]$. and Proof. Multiplying (25) by $t^{\tau-1} \hbar(m g(a) g(b) /(t / 2) g(b)+$ $(1-(t / 2))(g(a) / m)) E_{\sigma, \tau, \delta}^{\rho, r, k, c}\left(\omega t^{\sigma} ; p\right)$ and integrating over $[0,1]$, we get

$$
\begin{aligned}
& f\left(\frac{2 m^{2} g(a) g(b)}{g(a)+m g(b)}\right) \int_{0}^{1} t^{\tau-1} \hbar\left(\frac{m g(a) g(b)}{(t / 2) g(b)+(1-(t / 2))(g(a) / m)}\right) E_{\sigma, \tau, \delta}^{\rho, r, c}\left(\omega t^{\sigma} ; p\right) \mathrm{d} t \\
& \quad \leq h\left(\frac{1}{2^{\alpha}}\right) \int_{0}^{1} t^{\tau-1} E_{\sigma, \tau, \delta}^{\rho, r, c}\left(\omega t^{\sigma} ; p\right) \hbar\left(\frac{m g(a) g(b)}{(t / 2) g(b)+(1-(t / 2))(g(a) / m)}\right) f\left(\frac{m g(a) g(b)}{(t / 2) g(b)+(1-(t / 2))(g(a) / m)}\right) \mathrm{d} t \\
& \quad+m h\left(1-\frac{1}{2^{\alpha}}\right) \int_{0}^{1} t^{\tau-1} E_{\sigma, \tau, \delta}^{\rho, r, k, c}\left(\omega t^{\sigma} ; p\right) \hbar\left(\frac{m g(a) g(b)}{(t / 2) g(b)+(1-(t / 2))(g(a) / m)}\right) f\left(\frac{m g(b)}{(t / 2) g(a)+m(1-(t / 2)) g(b)}\right) \mathrm{d} t .
\end{aligned}
$$


By choosing $g(x)=(m g(a) g(b) /(t / 2) g(b)+(1-(t / 2))$ $(g(a) / m))$ in (30) and using (4) and (5) and the condition $f(1 / g(x))=f(1 /(1 / g(a))+(1 / m g(b))-m g(x))$, the first inequality of (29) can be obtained. On the contrary, by the harmonically $(\alpha, h-m)$-convexity of $f$, multiplying (27) by $t^{\tau-1} \hbar(m g(a) g(b) /(t / 2) g(b)+(1-(t / 2))(g(a) / m)) E_{\sigma, \tau, \delta}^{\rho, r, k, c}$ $\left(\omega t^{\sigma} ; p\right)$ and integrating over $[0,1]$, we get

$$
\begin{aligned}
& h\left(\frac{1}{2^{\alpha}}\right) \int_{0}^{1} t^{\tau-1} E_{\sigma, \tau, \delta}^{\rho, r, k, c}\left(\omega t^{\sigma} ; p\right) \hbar\left(\frac{m g(a) g(b)}{(t / 2) g(b)+(1-(t / 2))(g(a) / m)}\right) f\left(\frac{m g(a) g(b)}{(t / 2) g(b)+(1-(t / 2))(g(a) / m)}\right) \mathrm{d} t \\
& +m h\left(1-\frac{1}{2^{\alpha}}\right) \int_{0}^{1} t^{\tau-1} E_{\sigma, \tau, \delta}^{\rho, r, k, c}\left(\omega t^{\sigma} ; p\right) \hbar\left(\frac{m g(a) g(b)}{(t / 2) g(b)+(1-(t / 2))(g(a) / m)}\right) \times f\left(\frac{m g(a) g(b)}{(t / 2) g(a)+m(1-(t / 2)) g(b)}\right) \mathrm{d} t \\
& \leq m\left(h\left(\frac{1}{2^{\alpha}}\right) f\left(\frac{g(a)}{m^{2}}\right)+m h\left(1-\frac{1}{2^{\alpha}}\right) f(g(b))\right) \\
& \times \int_{0}^{1} t^{\tau-1} E_{\sigma, \tau, \delta}^{\rho, r, k, c}\left(\omega t^{\sigma} ; p\right) \hbar\left(\frac{m g(a) g(b)}{(t / 2) g(b)+(1-(t / 2))(g(a) / m)}\right) h\left(\left(\frac{t}{2}\right)^{\alpha}\right) \mathrm{d} t \\
& +\left(h\left(\frac{1}{2^{\alpha}}\right) f(g(b))+m h\left(1-\frac{1}{2^{\alpha}}\right) f(g(a))\right) \\
& \times \int_{0}^{1} t^{\tau-1} E_{\sigma, \tau, \delta}^{\rho, r, k, c}\left(\omega t^{\sigma} ; p\right) \hbar\left(\frac{m g(a) g(b)}{(t / 2) g(b)+(1-(t / 2))(g(a) / m)}\right) h\left(1-\left(\frac{t}{2}\right)^{\alpha}\right) \mathrm{d} t .
\end{aligned}
$$

By choosing $g(x)=(m g(a) g(b) /(t / 2) g(b)+(1-(t / 2))$ $(g(a) / m))$ in (31) and using (4) and (5) and the condition $f(1 / g(x))=f(1 /(1 / g(a))+(1 / m g(b))-m g(x))$, the second inequality of (29) can be obtained.

\section{Remark 5}

(i) By setting $p=0, \alpha=m=1, h(t)=t$, and $g=I$, Theorem 3.6 of [15] is obtained

(ii) By setting $\alpha=m=1, h(t)=t$, and $g=I$, Theorem 5 of [16] is obtained
2.1. Results for Harmonically $(h-m)$-Convex Functions. By setting $\alpha=1$ in Theorems 3-6, the results for harmonically $(h-m)$-convex functions are obtained as follows.

Theorem 7. Under the assumptions of Theorem 3, the following inequality holds for harmonically $(h-m)$-convex functions:

$$
\begin{aligned}
& f(\left.\frac{2 m^{2} g(a) g(b)}{g(a)+m g(b)}\right)\left(\mathscr{F}_{g^{-1}\left(1 / m^{2} g(b)\right), \tau}^{g^{-1}(1 / m(a))^{-}}\right)\left(m^{\sigma} \omega^{\prime}, 1\right) \\
& \leq h\left(\frac{1}{2}\right)\left\{\left(\mathscr{F}_{g^{-1}\left(1 / m^{2} g(b)\right), \tau}^{g^{-1}(1 / m g(a))^{-}}\right)\left(m^{\sigma} \omega^{\prime}, f \circ \psi\right)+m^{1-2 \tau}\left(\mathscr{F}_{g^{-1}(1 / g(a)), \tau}^{g^{-1}(1 / m g(b))^{+}}\right)\left(\omega^{\prime}, f \circ \psi\right)\right\} \\
& \leq h\left(\frac{1}{2}\right)\left(\frac{m g(b)-g(a)}{m^{2} g(a) g(b)}\right)^{\tau}\left\{m\left(f\left(\frac{g(a)}{m^{2}}\right)+m f(g(b))\right)\right. \\
&\left.\quad \times \int_{0}^{1} t^{\tau-1} E_{\sigma, \tau, \delta}^{\rho, r, k, c}\left(\omega\left(t^{\sigma}\right) ; p\right) h(t) \mathrm{d} t+(f(g(b))+m f(g(a))) \int_{0}^{1} t^{\tau-1} E_{\sigma, \tau, \delta}^{\rho, r, k, c}\left(\omega\left(t^{\sigma}\right) ; p\right) h(1-t) \mathrm{d} t\right\} .
\end{aligned}
$$

Theorem 8. Under the assumptions of Theorem 4, the following inequality holds for harmonically $(h-m)$-convex functions: 


$$
\begin{aligned}
& f\left(\frac{2 m^{2} g(a) g(b)}{g(a)+m g(b)}\right)\left(\mathscr{F}_{g^{-1}\left(1 / m^{2} g(b)\right), \tau}^{g^{-1}(1 / m g(a))^{-}}\right)\left(m^{\sigma} \omega^{\prime}, \hbar \circ \psi\right) \\
& \leq h\left(\frac{1}{2}\right)(1+m)\left(\mathscr{F}_{g^{-1}\left(1 / m^{2} g(b)\right), \tau}^{g^{-1}(1 / m g(a)-}\right)\left(m^{\sigma} \omega^{\prime},(\hbar \circ \psi)(f \circ \psi)\right) \\
& \leq h\left(\frac{1}{2}\right)\left(\frac{m g(b)-g(a)}{m^{2} g(a) g(b)}\right)^{\tau}\left\{m\left(f\left(\frac{(a)}{m^{2}}\right)+m f(g(b))\right)\right. \\
& \quad \times \int_{0}^{1} t^{\tau-1} E_{\sigma, \tau, \delta}^{\rho, r, k, c}\left(\omega\left(t^{\sigma}\right) ; p\right) \hbar\left(\frac{m g(a) g(b)}{t g(b)+(1-t)(g(a) / m)}\right) h(t) \mathrm{d} t \\
&\left.\quad+(f(g(b))+m f(g(a))) \int_{0}^{1} t^{\tau-1} E_{\sigma, \tau, \delta}^{\rho, r, k, c}\left(\omega\left(t^{\sigma}\right) ; p\right) \hbar\left(\frac{m g(a) g(b)}{\operatorname{tg}(b)+(1-t)(g(a) / m)}\right) h(1-t) \mathrm{d} t\right\} .
\end{aligned}
$$

Theorem 9. Under the assumptions of Theorem 5, the following inequality holds for harmonically $(h-m)$-convex functions:

$$
\begin{aligned}
& f\left(\frac{2 m^{2} g(a) g(b)}{g(a)+m g(b)}\right)\left(\mathscr{F}_{g^{-1}\left(1 / m^{2} g(b)\right), \tau}^{g^{-1}\left(g(a)+m g(b) / 2 m^{2} g(a) g(b)\right)^{-}}\right)\left((2 m)^{\sigma} \omega^{\prime}, 1\right) \\
& \quad \leq h\left(\frac{1}{2}\right)\left\{\left(\mathscr{F}_{g^{-1}\left(1 / m^{2} g(b)\right), \tau}^{g^{-1}\left(g(a)+m g(b) / 2 m^{2} g(a) g(b)\right)^{-}}\right)\left((2 m)^{\sigma} \omega^{\prime}, f \circ \psi\right)+m^{1-2 \tau}\left(\mathscr{F}_{g^{-1}(1 / g(a)), \tau}^{g^{-1}(g(a)+m g(b) / 2 m g(a) g(b))^{+}}\right)\left(2^{\sigma} \omega^{\prime}, f^{\circ} \psi\right)\right\} \\
& \quad \leq h\left(\frac{1}{2}\right)\left(\frac{m g(b)-g(a)}{2 m^{2} g(a) g(b)}\right)^{\tau}\left\{m\left(f\left(\frac{g(a)}{m^{2}}\right)+m f(g(b))\right) \int_{0}^{1} t^{\tau-1} E_{\sigma, \tau, \delta}^{\rho, r, k}\left(\omega\left(t^{\sigma}\right) ; p\right) h\left(\frac{t}{2}\right) \mathrm{d} t\right. \\
& \left.\quad+(f(g(b))+m f(g(a))) \int_{0}^{1} t^{\tau-1} E_{\sigma, \tau, \delta}^{\rho, r, k}\left(\omega\left(t^{\sigma}\right) ; p\right) h\left(1-\frac{t}{2}\right) \mathrm{d} t\right\} .
\end{aligned}
$$

Theorem 10. Under the assumptions of Theorem 6, the following inequality holds for harmonically $(h-m)$-convex functions:

$$
\begin{aligned}
& f\left(\frac{2 m^{2} g(a) g(b)}{g(a)+m g(b)}\right)\left(\mathscr{F}_{g^{-1}\left(1 / m^{2} g(b)\right), \tau}^{g^{-1}\left(g(a)+m g(b) / 2 m^{2} g(a) g(b)\right)^{-}}\right)\left((2 m)^{\sigma} \omega^{\prime}, \hbar \circ \psi\right) \\
& \leq h\left(\frac{1}{2}\right)(1+m)\left(\mathscr{F}_{g^{-1}\left(1 / m^{2} g(b)\right), \tau}^{g^{-1}\left(g(a)+m g(b) / 2 m^{2} g(a) g(b)\right)^{-}}\right)\left((2 m)^{\sigma} \omega^{\prime},(\hbar \circ \psi)(f \circ \psi)\right) \\
& \leq h\left(\frac{1}{2}\right)\left(\frac{m g(b)-g(a)}{2 m^{2} g(a) g(b)}\right)^{\tau}\left\{m\left(f\left(\frac{g(a)}{m^{2}}\right)+m f(g(b))\right)\right. \\
& \quad \times \int_{0}^{1} t^{\tau-1} E_{\sigma, \tau, \delta}^{\rho, r, k, c}\left(\omega\left(t^{\sigma}\right) ; p\right) \hbar\left(\frac{m g(a) g(b)}{(t / 2) g(b(1-(t / 2)))+(g(a) / m)}\right) h\left(\frac{t}{2}\right) \mathrm{d} t m g(a) g(b) \\
&\left.\quad+(f(g(b))+m f(g(a))) \int_{0}^{1} t^{\tau-1} E_{\sigma, \tau, \delta}^{\rho, r, k, c}\left(\omega\left(t^{\sigma}\right) ; p\right) \hbar\left(\frac{t}{(t / 2) g(b)+(1-(t / 2))(g(a) / m)}\right) h\left(1-\frac{t}{2}\right) \mathrm{d} t\right\} .
\end{aligned}
$$


2.2. Results for Harmonically $(\alpha, m)$-Convex Functions. By setting $h(t)=t$ in Theorems $3-6$, the results for harmonically $(\alpha-m)$-convex functions are obtained as follows.
Theorem 11. Under the assumptions of Theorem 3, the following inequality holds for harmonically $(\alpha, m)$-convex functions:

$$
\begin{aligned}
& f\left(\frac{2 m^{2} g(a) g(b)}{g(a)+m g(b)}\right)\left(\mathscr{F}^{g^{-1}(1 / m g(a))^{-}}\right)\left(m^{\sigma} \omega^{\prime}, 1\right) \\
& \leq\left(\frac{1}{2^{\alpha}}\right)\left\{\left(\mathscr{F}_{g^{-1}\left(1 / m^{2} g(b)\right), \tau}^{g^{-1}(1 / m g(a))^{-}}\right)\left(m^{\sigma} \omega^{\prime}, f \circ \psi\right)+m^{1-2 \tau}\left(2^{\alpha}-1\right)\left(\mathscr{F}_{g^{-1}(1 / g(a)), \tau}^{g^{-1}(1 / m g(b))^{+}}\right)\left(\omega^{\prime}, f \circ \psi\right)\right\} \\
& \leq\left(\frac{1}{2^{\alpha}}\right)\left(\frac{m g(b)-g(a)}{m^{2} g(a) g(b)}\right)^{\tau}\left\{m\left(f\left(\frac{g(a)}{m^{2}}\right)+m\left(2^{\alpha}-1\right) f(g(b))\right)\right. \\
& \times\left(\begin{array}{c}
\mathscr{F}^{g^{-1}(1 / m g(a))^{-}} \\
g^{-1}\left(1 / m^{2} g(b)\right), \tau
\end{array}\right)\left(m^{\sigma} \omega^{\prime}, 1\right)+\left(f(g(b))+m\left(2^{\alpha}-1\right) f(g(a))\right) \\
& \left.\times\left(\left(\mathscr{F}_{g^{-1}\left(1 / m^{2} g(b)\right), \tau}^{g^{-1}(1 / m g(a))^{-}}\right)\left(m^{\sigma} \omega^{\prime}, 1\right)-\left(\begin{array}{c}
\mathscr{F}^{g^{-1}(1 / m g(a))^{-}} \\
g^{-1}\left(1 / m^{2} g(b)\right), \tau+\alpha
\end{array}\right)\left(m^{\sigma} \omega^{\prime}, 1\right)\right)\right\} .
\end{aligned}
$$

Theorem 12. Under the assumptions of Theorem 4, the following inequality holds for harmonically $(\alpha, m)$-convex functions:

$$
\begin{aligned}
& f\left(\frac{2 m^{2} g(a) g(b)}{g(a)+m g(b)}\right)\left(\mathscr{F}_{g^{-1}(1 / m g(a))^{-}}\right)\left(m^{\sigma} \omega^{\prime}, \hbar \circ \psi\right) \\
& \leq\left(\frac{1}{2^{\alpha}}\right)(1+m)\left(\begin{array}{c}
\mathscr{F}^{g^{-1}(1 / m g(a))^{-}} \\
g^{-1}\left(1 / m^{2} g(b)\right), \tau
\end{array}\right)\left(m^{\sigma} \omega^{\prime},(\hbar \circ \psi)(f \circ \psi)\right) \\
& \leq\left(\frac{1}{2^{\alpha}}\right)\left(\frac{m g(b)-g(a)}{m^{2} g(a) g(b)}\right)^{\tau}\left\{m\left(f\left(\frac{g(a)}{m^{2}}\right)+m\left(2^{\alpha}-1\right) f(g(b))\right)\right. \\
& \times \int_{0}^{1} t^{\tau+\alpha-1} E_{\sigma, \tau, \delta}^{\rho, r, k, c}\left(\omega\left(t^{\sigma}\right) ; p\right) \hbar\left(\frac{m g(a) g(b)}{\operatorname{tg}(b)+(1-t)(g(a) / m)}\right) \mathrm{d} t+\left(f(g(b))+m\left(2^{\alpha}-1\right) f(g(a))\right) \\
& \left.\times \int_{0}^{1}\left(t^{\tau-1}-t^{\tau+\alpha-1}\right) E_{\sigma, \tau, \delta}^{\rho, r, k, c}\left(\omega\left(t^{\sigma}\right) ; p\right) \hbar\left(\frac{m g(a) g(b)}{\operatorname{tg}(b)+(1-t)(g(a) / m)}\right) \mathrm{d} t\right\} .
\end{aligned}
$$

Theorem 13. Under the assumptions of Theorem 5, the following inequality holds for harmonically $(\alpha, m)$-convex functions:

$$
\begin{aligned}
f( & \left.\frac{2 m^{2} g(a) g(b)}{g(a)+m g(b)}\right)\left(\mathscr{F}_{g^{-1}\left(1 / m^{2} g(b)\right), \tau}^{g^{-1}\left(g(a)+m g(b) / 2 m^{2} g(a) g(b)\right)^{-}}\right)\left((2 m)^{\sigma} \omega^{\prime}, 1\right) \\
\leq & \left(\frac{1}{2^{\alpha}}\right)\left\{\left(\mathscr{F}_{g^{-1}\left(1 / m^{2} g(b)\right), \tau}^{g^{-1}\left(g(a)+m g(b) / 2 m^{2} g(a) g(b)\right)^{-}}\right)\left((2 m)^{\sigma} \omega^{\prime}, f \circ \psi\right)+m^{1-2 \tau}\left(\mathscr{F}_{g^{-1}(1 / g(a)), \tau}^{g^{-1}(g(a)+m g(b) / 2 m g(a) g(b))^{+}}\right)\left(2^{\sigma} \omega^{\prime}, f \circ \psi\right)\right\} \\
\leq & \left(\frac{1}{2^{\alpha}}\right)\left(\frac{m g(b)-g(a)}{2 m^{2} g(a) g(b)}\right)^{\tau}\left\{m\left(f\left(\frac{g(a)}{m^{2}}\right)+m\left(2^{\alpha}-1\right) f(g(b))\right)\right. \\
& \times\left(\frac{1}{2^{\alpha}}\right)\left(\begin{array}{c}
\mathscr{F}^{-1}\left(g(a)+m g(b) / 2 m^{2} g(a) g(b)\right)^{-} \\
g^{-1}\left(1 / m^{2} g(b)\right), \tau+\alpha
\end{array}\right)\left((2 m)^{\sigma} \omega^{\prime}, 1\right)+\left(f(g(b))+m\left(2^{\alpha}-1\right) f(g(a))\right) \\
& \left.\times\left(\left(\mathscr{F}_{g^{-1}\left(1 / m^{2} g(b)\right), \tau}^{g^{-1}\left(g(a)+m g(b) / 2 m^{2} g(a) g(b)\right)^{-}}\right)\left((2 m)^{\sigma} \omega^{\prime}, 1\right)-\left(\frac{1}{2^{\alpha}}\right)\left(\mathscr{F}_{g^{-1}\left(1 / m^{2} g(b)\right), \tau+\alpha}^{g^{-1}\left(g(a)+m g(b) / 2 m^{2} g(a) g(b)\right)^{-}}\right)\left((2 m)^{\sigma} \omega^{\prime}, 1\right)\right)\right\} .
\end{aligned}
$$


Theorem 14. Under the assumptions of Theorem 6, the following inequality holds for harmonically $(\alpha, m)$-convex functions:

$$
\begin{aligned}
& f\left(\frac{2 m^{2} g(a) g(b)}{g(a)+m g(b)}\right)\left(\mathscr{F}_{g^{-1}\left(1 / m^{2} g(b)\right), \tau}^{\left.g^{-1}\left(g(a)+m g(b) / 2 m^{2} g(a) g(b)\right)^{-}\right)\left((2 m)^{\sigma} \omega^{\prime}, \hbar \circ \psi\right)}\right. \\
& \leq\left(\frac{1}{2^{\alpha}}\right)(1+m)\left(\mathscr{F}_{g^{-1}\left(1 / m^{2} g(b)\right), \tau}^{g^{-1}\left(g(a)+m g(b) / 2 m^{2} g(a) g(b)\right)^{-}}\right)\left((2 m)^{\sigma} \omega^{\prime},(\hbar \circ \psi)(f \circ \psi)\right) \\
& \leq\left(\frac{1}{2^{\alpha}}\right)\left(\frac{m g(b)-g(a)}{2 m^{2} g(a) g(b)}\right)^{\tau}\left\{m\left(f\left(\frac{g(a)}{m^{2}}\right)+m\left(2^{\alpha}-1\right) f(g(b))\right)\right. \\
& \quad \times \int_{0}^{1} t^{\tau-1} E_{\sigma, \tau, \delta}^{\rho, r, k, c}\left(\omega\left(t^{\sigma}\right) ; p\right) \hbar\left(\frac{m g(a) g(b)}{(t / 2) g(b)+(1-(t / 2))(g(a) / m)}\right)\left(\frac{t}{2}\right)^{\alpha} \mathrm{d} t \\
& \quad+\left(f(g(b))+m\left(2^{\alpha}-1\right) f(g(a))\right) \\
& \left.\quad \times \int_{0}^{1} t^{\tau-1} E_{\sigma, \tau, \delta}^{\rho, r, k, c}\left(\omega\left(t^{\sigma}\right) ; p\right) \hbar\left(\frac{m g(a) g(b)}{(t / 2) g(b)+(1-(t / 2))(g(a) / m)}\right)\left(1-\left(\frac{t}{2}\right)^{\alpha}\right) \mathrm{d} t\right\} .
\end{aligned}
$$

\section{Concluding Remarks}

The presented work includes two generalized fractional versions of the Hadamard inequality (stated in Theorems 3 and5) as well as two generalized fractional versions of the Fejér-Hadamard inequality (stated in Theorems 4 and6) for a new generalized harmonic convexity. Their connections with already published results are shown in Remarks 2, 3, 4, and 5. Also, we work out these inequalities for harmonically $(\alpha, m)$-convex and $(h-m)$-convex functions. The reader can obtain a series of Hadamard and Fejér-Hadamard inequalities for fractional integral operators containing Mittag-Leffler functions for several kinds of convex functions.

\section{Data Availability}

No data were used to support the findings of this study.

\section{Conflicts of Interest}

The authors declare that they have no conflicts of interest.

\section{Acknowledgments}

This research was supported by the National Natural Science Foundation of China (Grant nos. 11971142, 11871202, $61673169,11701176,11626101$, and 11601485).

\section{References}

[1] B. Ahmad, A. Alsaedi, M. Kirane, and B. T. Torebek, "Hermite-Hadamard, Hermite-Hadamard-Fejér, Dragomir-Agarwal and pachpatte type inequalities for convex functions via new fractional integrals," Journal of Computational and Applied Mathematics, vol. 353, pp. 120-129, 2019.
[2] M. Andrić, G. Farid, and J. Pečarić, "A further extension of Mittag-Leffler function," Fractional Calculus and Applied Analysis, vol. 21, no. 5, pp. 1377-1395, 2018.

[3] H. Chen and U. N. Katugampola, "Hermite-Hadamard and Hermite-Hadamard-Fejér type inequalities for generalized fractional integrals," Journal of Mathematical Analysis and Applications, vol. 446, no. 2, pp. 1274-1291, 2017.

[4] F. Chen and S. Wu, "Fejér and Hermite-Hadamard type inequalities for harmonically convex functions," Journal of Applied Mathematics, vol. 2014, pp. 1-6, Article ID 386806, 2014.

[5] Z. Dahmani, "On Minkowski and Hermite-Hadamard integral inequalities via fractional integration," Annals of Functional Analysis, vol. 1, no. 1, pp. 51-58, 2010.

[6] I. Iscan and S. Wu, "Hemite-Hadamard type inequalities for harmonically convex functions via fractional integrals," Applied Mathematics and Computation, vol. 238, pp. 237-244, 2014.

[7] X. Qiang, G. Farid, M. Yussouf, K. A. Khan, and A. Ur Rehman, "New generalized fractional versions of Hadamard and Fejér inequalities for harmonically convex functions," Journal of Inequalities and Applications, vol. 2020, no. 1, 2020.

[8] S. Kang, G. Abbas, G. Farid, and W. Nazeer, "A generalized Fejér-Hadamard inequality for harmonically convex functions via generalized fractional integral operator and related results," Mathematics, vol. 6, no. 7, p. 122, 2018.

[9] G. Farid, "A unified integral operator and further its consequences," Open Journal of Mathematical Analysis, vol. 4, no. 1, pp. 1-7, 2020.

[10] L. Fejér, "Überdie Fourierreihen II," Math Naturwiss Anz Ungar Akad Wiss, vol. 24, pp. 369-390, 1906.

[11] I. Iscan, "Hermite-Hadamard type inequalities for harmonically convex functions," Hacettepe Journal of Mathematics and Statistics, vol. 43, no. 6, pp. 935-942, 2014.

[12] I. Iscan, "Hermite-Hadamard type inequalities for harmonically $(\alpha, m)$-convex functions," https://arxiv.org/abs/1307. 5402. 
[13] M. A. Noor, K. I. Noor, M. U. Awan, and S. Costaache, "Some integral inequalities for harmonically $h$-convex functions," UPB Scientific Bulletin, Series A, vol. 77, no. 1, pp. 5-16, 2015.

[14] I. A. Baloch and İ. İşcan, "Some Ostrowski type inequalities for harmonically s, m-convex functions in second sense," International Journal of Analysis, vol. 2015, pp. 1-9, Article ID 672675, 2015.

[15] G. Abbas and G. Farid, "Hadamard and Fejér-Hadamard type inequalities for harmonically convex functions via generalized fractional integrals," The Journal of Analysis, vol. 25, no. 1, pp. 107-119, 2017.

[16] G. Farid, A. UR. Rehman, and S. Mehmood, "Hadamard and Fejér-Hadamard type integral inequalities for harmonically convex functions via an extended generalized Mittag-Leffler function," Journal of Mathematical and Computational Science, vol. 8, no. 5, pp. 630-643, 2018.

[17] M. Kunt, I. Iscan, N. Yazi, and U. Gozutok, "On new inequalities of Hermite-Hadamard-Fejér type inequalities for harmonically convex functions via fractional integrals," Springer Plus, vol. 5, no. 1, pp. 1-19, 2016. 